\title{
Denver County Clostridium difficile Trends and Associated Risk Factors 2011-2013
}

\author{
Anna D. Oberste ${ }^{\star 1}$, Kathryn H. DeYoung ${ }^{1}$, Helen Johnston ${ }^{2}$, Stephanie Gravitz ${ }^{1}$, Emily \\ McCormick ${ }^{1}$ and Arthur Davidson ${ }^{1}$
}

${ }^{1}$ Epidemology-Preparedness, Denver Public Health, Denver, CO, USA; ${ }^{2}$ Colorado Department of Public Health and Environment, Denver, CO, USA

\section{Objective}

Identify population-based Clostridium difficile infection (CDI) incidence stratified by Health Care Facility Onset (HCFO), Community Onset-Healthcare Facility Associated (CO-HCFA), and Community Onset-Community Associated (CO-CA) CDI in Denver County from 2011 - 2013 and describe demographic, health care facility exposure, and medication use risk factors.

\section{Introduction}

Clostridium difficile (CD), a gram-negative, anaerobic, sporeforming bacterium causes symptoms ranging from mild to severe diarrhea and may result in death. ${ }^{1}$ Approximately $75 \%$ of CDI cases have symptom onset outside of health care settings. ${ }^{2}$ Annual US costs of treatment and infection containment have surpassed $\$ 4.8$ billion. ${ }^{3}$ Risk factors for CDI include recent broad-spectrum antibiotic exposure, advanced age, severe underlying morbidities, immunocompromised status, long-term hospital stays, and residence in long-term-care facilities. ${ }^{4}$ Nationally, CO-CA cases have increased from $2.8 / 100,000$ person in 1993 to $14.9 / 100,000$ person in $2005 .^{5}$

\section{Methods}

A retrospective chart review studied $C D I$ cases from 2011 through 2013 with incident (no positive test in the previous 8 weeks) stool specimen (CDI positive toxin or molecular assay) reported to the state health department, among Denver County residents older than 1 year. Cases were randomly selected for chart review. Cases were stratified by epidemiologic classification (HFCO, CO-HCFA or CO-CA) and analyzed for associations with: age, gender, antibiotic exposure, underlying disease, and exposure to high risk areas (e.g., long-term care facilities and hospitals). Rates were calculated using State Demography Office-Department of Local Affairs information. Descriptive statistics (means and frequencies) were used to describe CDI trends by year, demographic group, epidemiologic classification, and risk factors.

\section{Results}

Between 2011 and 2013, 2503 CDI cases were reported and 892 cases were chart reviewed (22\%) and identified as CO-CA (339), COHCFA (175), or HCFO (44). Denver CDI incidence rate increased from 129/100,000 residents in 2010 to 139/100,000 residents in 2013. Incidence rates of cases classified as HCFO and CO-HCFA remained stable over the study period. Rates of CO-CA cases decreased from $50.4 / 100,000$ residents in 2011 to 46/100,000 residents in 2013. The mean age for $\mathrm{HCFO}$ cases was 67 years, 49 years for CO-CA cases, and 56 years for CO-HCFA cases. HCFO was more common among men; there were no gender disparities for CO-CA or CO-HCFA. During the study period, the presence of underlying morbidities increased in all onset types with over half of CO-CA and CO-HCFA cases reporting underlying morbidities in 2013 (CO-CA: 65\%; COHCFA: 69\%). Antibiotic exposure substantially increased between 2011 and 2013, in cases classified as CO-HCFA (29\% to 70\%), and CO-CA $(18 \%$ to $50 \%)$.

\section{Conclusions}

Rates of CO-CA CDI rose during the study period. Our study reflects national trends in age and antibiotic exposure by epidemiologic classification. ${ }^{6}$ The frequency of antibiotic exposure and underlying morbidities increased from 2011 to 2013 in both CO-CA and COHCFA cases. Denver CO-CA cases had more underlying morbidities compared to recent reports. ${ }^{?}$

\section{Keywords}

Clostridium difficile; healthcare associated infections; community acquired infections; Infectious disease

\section{References}

1. Halabi WJ, Nguyen VQ, Carmichael JC, et al. Clostridium difficile colitis in the United States: a decade of trends, outcomes, risk factors for colectomy, and mortality after colectomy. J. Am. Coll. Surg. Nov 2013;217(5):802-812.

2. Centers for Disease Control and Prevention (CDC). Vital Signs: Preventing Clostridium difficile Infections. Morb. Mortal. Weekly Rep. 2012;61(09):157-162.

3. Dubberke ER, Olsen MA. Burden of Clostridium difficile on the healthcare system. Clin Inf Dis . Aug 2012;55 Suppl 2:S88-92.

4. Dubberke E. Strategies for prevention of Clostridium difficile infection. J. Hosp. Med. Mar 2012;7 Suppl 3:S14-17.

5. Evans C., Safdar N. Current Trends in the Epidemiology and outcomes of Clostridium difficile infection. Clin Inf Dis 2015 (60): S66-71

6. Chitnis $\mathrm{AS}^{1}$, Holzbauer SM, Belflower RM, et al. Epidemiology of community-associated Clostridium difficile infection, 2009 through 2011 JAMA Intern Med. 2013 Jul 22;173(14):1359-67.

7. Dumyati, G., Stevens, V., Hannet G.E., Community -associated Clostridium difficile Infections, Monroe, County, New York, USA. Emerg Inf Dis. 2012 Mar; 18(3): 392-400.

*Anna D. Oberste

E-mail: Anna.Oberste@dhha.org 